\title{
Synthesis of some benzimidazole derivatives endowed with 1,2,3-triazole as potential inhibitors of hepatitis $C$ virus
}

\author{
BAHAA G. M. YOUSSIF ${ }^{1,2}$ \\ YASEEN A. M. MOHAMED 3 \\ MOHAMMED T. A. SALIM ${ }^{4}$ \\ FUYUHIKO INAGAKI ${ }^{5}$ \\ CHISATO MUKAI ${ }^{5}$ \\ HAJJAJ H. M. ABDU-ALLAH ${ }^{1 *}$ \\ ${ }^{1}$ Pharmaceutical Organic Chemistry \\ Department, Faculty of Pharmacy \\ Assiut University, Assiut, 71526, Egypt \\ ${ }^{2}$ Pharmaceutical Chemistry Department \\ College of Pharmacy, Al Jouf University \\ AlJouf Sakaka-2014, Kingdom of Saudi \\ Arabia \\ ${ }^{3}$ Pharmaceutical Organic Chemistry \\ Department, Faculty of Pharmacy \\ Al-Azhar University, Assiut Branch, Egypt \\ ${ }^{4}$ Microbiology and Immunology \\ Department, Faculty of Pharmacy \\ Al-Azhar University, Assiut Branch, Egypt \\ ${ }^{5}$ Division of Pharmaceutical Science \\ Graduate School of Natural Science and \\ Technology, Kanazawa University \\ Kakuma-machi, Kanazawa 920-1192, Japan
}

\begin{abstract}
New derivatives of 2-thiobenzimidazole incorporating triazole moiety were synthesized, characterized and tested in vitro for antiviral activity against hepatitis $\mathrm{C}$ virus (HCV) and hepatitis B virus (HBV). Their cytotoxicity was determined by the reduction in the number of viable cell. All of the synthesized compounds are inactive against $\mathrm{HBV}$ and some showed activity against HCV. In particular, two compounds showed significant activity, 2-\{4-[(1-benzoylbenzimidazol-2-ylthio)methyl]-1H-1,2,3-triazol-1-yl\}- $N$-( $p$-nitro-phenyl)-acetamide (13) and 2-(4-\{[1-( $p$-chlorobenzoyl)-benzimidazol-2-ylthio)methyl]-1H-1,2,3-triazol-1-yl\}- $N$-( $p$-nitrophenyl)-acetamide (17). The results give an insight into the importance of the substituent at position 2 of benzimidazole for the inhibition of $\mathrm{HCV}$.
\end{abstract}

Keywords: benzimidazole, triazole, anilide, antiviral, HCV, HBV

Accepted October 27, 2015

Published online February 25, 2016

Hepatitis C virus (HCV), a member of the Hepacvirus genus of the family Flavivridae, is a major etiological agent of human liver disease. HCV has infected an estimated 170 million people worldwide. HCV infection is often asymptomatic; however, it frequently causes chronic hepatitis, which progresses to the end-stage liver diseases, such as liver cirrhosis and hepatocellular carcinoma. For a long time, the therapeutic combination of pegylated alpha interferon (peg-IFN) and the nucleoside analogue ribavirin were used for

\footnotetext{
*Correspondence; e-mail: hajjajabduallah@yahoo.com; hagag.abdallah@pharm.au.edu.eg
} 
HCV treatment and were found to have a sustained viral response (SVR) rate of $\sim 50 \%$ in genotype 1 and 4 of HCV-infected patients (1). Recently, a new anti-HCV agent (Sofosbu$\operatorname{vir}^{R}$ ) was added to the above mentioned combination and produced improvement in the SVR up to $\sim 90 \%$ (1). However, peg-IFN and ribavirin should be included for successful treatment, particularly in the treatment of genotype 4. Combined therapy of HCV infections with peg-IFN is associated with serious side effects such as depression, flu-like symptoms, fatigue and hemolytic anemia caused by ribavirin $(2,3)$.

Therefore, finding a single compound for the treatment of HCV that promises to cure the majority of patients without the complications of combined therapy and/or the need for painful injections would be a good achievement.

Owing to its isosterism with indole and purine nuclei present in many fundamental cellular components and bioactive compounds, the benzimidazole ring represents a kind of privileged substructure from which several important drugs used in different therapeutic areas have been obtained, especially antitumor and antiviral agents. Among the antiviral benzimidazoles, an important position is held by compounds acting against HIV-1, $\mathrm{HCV}$ and respiratory syncytial virus (RSV) $(4,5)$.

The benzimidazole scaffold is common in different anti-HCV agents with different modes of action. Derivatives of bis-benzimidazolemethane were discovered to be highly potent, reversible and selective serine protease inhibitors $(6,7)$. Furthermore, by replacing the 2-methylbenzimidazole moiety of this compound with 2-pyrimidomethylenethio moiety or its bioisosteric 2-pyrimidomethyleneamino group was shown to have a positive impact on the inhibitory activity against HCV (8). Moreover, several 2-substituted benzimidazoles showed good antiviral activity $(9,10)$.

The antiviral activity was found in all the subsets of benzimidazole derivatives, but it was not evenly distributed among them. The activity is a result of several interacting structural features, which may direct it $v$ s. specific viruses, modulating also their potency (11).

On the other hand, the 1,2,3-triazole core has been applied in many synthetic approaches. Several compounds containing this heterocycle exhibited a broad range of biological activities including against HIV and HCV (12-15).

Considering the importance of 2-substituted benzimidazole and triazole nucleus, it was thought worthwhile to design and synthesize some new 2-substituted benzimidazole derivatives connected to differently substituted anilides with triazole moiety (Fig. 1) and to screen them for their potential antiviral activity. This approach may provide scaffolds in which pharmacophores can be arranged so as to yield potent and hopefully selective inhibitors.

\section{EXPERIMENTAL}

\section{Chemistry}

Reagents and solvents. - All reagents and solvents were obtained from commercial suppliers and were used without further purification. The starting materials, 2-(prop-2-ynylthio)- $1 H$-benzimidazole $(1,16), 2$-azido- $N$-phenylacetamide (3a), 2-azido- $N$ - $(p$-chlorophenyl)acetamide (3b), 2-azido- $N$-( $p$-bromophenyl)acetamide (3c), 2-azido- $N$-( $p$-methylphenyl) 

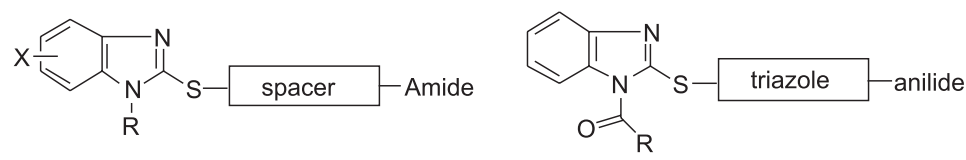

$\mathrm{R}=\mathrm{H}$, sulfonamide carbamate

spacer $=$ no spacer or heterocycles

$\mathrm{X}=\mathrm{H}$, halide or alkoxy

$\mathrm{R}=$ alkyl group

the main structural features of

the reported benzimidazoles with antiviral activity (references 8-15)

Fig. 1. Rationale for the design of target compounds 4-18.

acetamide (3d) and 2-azido- $N$-( $p$-nitrophenyl)acetamide (3e) were prepared according to reported procedures (17).

Procedures. - Melting points are uncorrected and were determined on an electrothermal melting point apparatus (Stuart Scientific, model SMP3, UK). Precoated silica gel plates (Kieselgel $0.25 \mathrm{~mm}$, 60G F254, Merck, Germany) were used for TLC monitoring of the reactions. The developing solvent system $\mathrm{CH}_{2} \mathrm{Cl}_{2} / \mathrm{CH}_{3} \mathrm{OH}(9.5: 0.5, \mathrm{~V} / \mathrm{V})$ was used and the spots were detected at $254 \mathrm{~nm}$ using an ultraviolet lamp (model CM-10, Spectroline, USA).

IR spectra (KBr discs) were recorded on a Shimadzu IR-470 spectrometer (Shimadzu, Japan). ${ }^{1} \mathrm{H}(500 \mathrm{MHz})$ and ${ }^{13} \mathrm{C}$ NMR $(125 \mathrm{MHz})$ spectra were obtained with JEOL JNM-ECA 500 spectrometer (JEOL, Japan) with tetramethylsilane (TMS) as an internal standard. Chemical shifts were expressed in $\delta$-value (ppm) relative to TMS. DMSO- $d_{6}$ was used as a solvent. Addition of deuterium oxide was used for the detection of exchangeable protons. Mass spectra were recorded with a JEOL JMS600 mass spectrometer.

2-Acyl-1-propargyl-thiobenzimidazoles $(2 a-c)$. General procedure. - To a suspension of 2-(prop-2-ynyl-thio)-1H-benzimidazole (1) (188 mg, $1 \mathrm{mmol}$ ) (16) and potassium carbonate anhydrous (145 mg, $1.05 \mathrm{mmol}, 1.05$ equivalent) in dry acetone $(10 \mathrm{~mL})$, the appropriate acyl halide (1.05 equivalent) was added. The reaction mixture was stirred for $12-18 \mathrm{~h}$ at ambient temperature. Acetone was evaporated, the residue was treated with water and then extracted with $\mathrm{CH}_{2} \mathrm{Cl}_{2}(3 \times 10 \mathrm{~mL})$. The organic extract was washed with water, dried over anhydrous $\mathrm{Na}_{2} \mathrm{SO}_{4}$ and then concentrated. The obtained crude products were recrystallized from $\mathrm{DMF} / \mathrm{H}_{2} \mathrm{O}$ (7:3).

2-\{4-[(1-Acylbenzimidazol-2-yl-thio)methyl]-1H-1,2,3-triazol-1-yl\}-N-(unsubstituted/substituted phenylacetamides (4-18). General procedure. - To a solution of propargyl derivatives 1 or 2a-c $(1.3 \mathrm{mmol})$ in a mixture of $\mathrm{THF} / \mathrm{H}_{2} \mathrm{O}(12 \mathrm{~mL}, 2: 1)$, the reported azide derivatives 3a-e (17) $(1.6 \mathrm{mmol})$, copper sulphate $(0.13 \mathrm{mmol})$ and sodium ascorbate $(0.26 \mathrm{mmol})$ were added. The resulting mixture was stirred at room temperature for $20 \mathrm{~h}$, and the residue was extracted with $\mathrm{CH}_{2} \mathrm{Cl}_{2}(3 \times 15 \mathrm{~mL})$. The organic phase was washed with water, dried over anhydrous $\mathrm{Na}_{2} \mathrm{SO}_{4}$ and concentrated. The crude product was purified by column chromatography using $\mathrm{CH}_{2} \mathrm{Cl}_{2} /$ methanol (9:1) as an eluent to give pure products (4-18).

Physical and spectral data of the newly synthesized compounds (2a-c and 4-18) are collected in Tables I and II. 
B. G. M. Youssif et al.: Synthesis of some benzimidazole derivatives endowed with 1,2,3-triazole as potential inhibitors of hepatitis C virus, Acta Pharm. 66 (2016) 219-231.

Table I. Physical and ESI-HRMS data of the newly synthesized compounds

\begin{tabular}{lcccc}
\hline Compound & M. p. & Yield & Mol. & ESI-HRM \\
$\left({ }^{\circ} \mathrm{C}\right)$ & $(\%)$ & formula & (calcd./found $)$
\end{tabular}

2a 1-Acetyl-2-propyn-2-ylthiobenzimidazole

163-165 $86 \quad \mathrm{C}_{12} \mathrm{H}_{11} \mathrm{~N}_{2} \mathrm{OS}$

231.0592

2b 1-Benzoyl-2-propyn-2-ylthiobenzimidazole

(13)

2b 1-Benzoyl-2-propyn-2-ylthiobenzimidazole

131-132 $81 \quad \mathrm{C}_{17} \mathrm{H}_{13} \mathrm{~N}_{2} \mathrm{OS}$

231.0586

2c $\begin{aligned} & \text { 1-p-Chlorobenzoyl-2-propyn-2-ylthiobenzimid- } \\ & \text { azole }\end{aligned}$

129-130 $72 \quad \mathrm{C}_{17} \mathrm{H}_{12} \mathrm{ClN}_{2} \mathrm{OS}$

293.0749

293.0753

4 2-\{4-[(1-Acetylbenzimidazol-2-ylthio)methyl]- $1 H$ -

4 1,2,3-triazol-1-yl\}-N-phenylacetamide

196-198 $89 \quad \mathrm{C}_{20} \mathrm{H}_{19} \mathrm{~N}_{6} \mathrm{O}_{2} \mathrm{~S}$

327.0359

327.0365

5 2-\{4-[(1-Acetylbenzimidazol-2-ylthio)methyl]- $1 H$ -

5 1,2,3-triazol-1-yl\}-N-( $p$-chlorophenyl)-acetamide

201-203 $93 \quad \mathrm{C}_{20} \mathrm{H}_{18} \mathrm{ClN}_{6} \mathrm{O}_{2} \mathrm{~S}$

407.1297

407.1290

$6 \begin{aligned} & \text { 2-\{4-[(1-Acetylbenzimidazol-2-ylthio)methyl]- } 1 H \text { - } \\ & \text { 1,2,3-triazol-1-yl\}- } N \text {-( } p \text {-bromophenyl)-acetamide }\end{aligned}$

$$
\text { - }
$$

7 2-\{4-[(1-Acetylbenzimidazol-2-ylthio)methyl]- $1 H$ -

7 1,2,3-triazol-1-yl\}-N-( $p$-methylphenyl)-acetamide

8 2-\{4-[(1-Acetylbenzimidazol-2-ylthio)methyl]-1H1,2,3-triazol-1-yl\}-N-(p-nitrophenyl)-acetamide

195-197 87

202-204

83

$\mathrm{C}_{20} \mathrm{H}_{18} \mathrm{BrN}_{6} \mathrm{O}_{2} \mathrm{~S}$

441.0900

441.0896

485.0395

485.0391

207-209 $88 \quad \mathrm{C}_{21} \mathrm{H}_{21} \mathrm{~N}_{6} \mathrm{O}_{2} \mathrm{~S}$

421.1447

421.1451

452.1141

452.1141

9 2-\{4-[(1-Benzoylbenzimidazol-2-ylthio)methyl]$1 H$-1,2,3-triazol-1-yl\}-N-phenylacetamide

198-199 91

$\mathrm{C}_{20} \mathrm{H}_{18} \mathrm{~N}_{7} \mathrm{O}_{4} \mathrm{~S}$

469.1447

469.1450

10 2-\{4-[(1-Benzoylbenzimidazol-2-ylthio)methyl]- $1 H$ -

10 1,2,3-triazol-1-yl\}-N-(p-chlorophenyl)acetamide

199-201 90

$\mathrm{C}_{25} \mathrm{H}_{21} \mathrm{~N}_{6} \mathrm{O}_{2} \mathrm{~S}$

503.1057

503.1060

11 2-\{4-[(1-Benzoylbenzimidazol-2-ylthio)methyl] $-1 H$ -

1,2,3-triazol-1-yl\}-N-( $p$-bromophenyl)-acetamide

206-208 89

$\mathrm{C}_{25} \mathrm{H}_{20} \mathrm{ClN}_{6} \mathrm{O}_{2} \mathrm{~S}$

547.0552

547.0544

12 2-\{4-[(1-Benzoylbenzimidazol-2-ylthio)methyl]- $1 H$ -

1,2,3-triazol-1-yl\}-N-( $p$-methylphenyl)-acetamide

197-199 89

$\mathrm{C}_{25} \mathrm{H}_{20} \mathrm{BrN}_{6} \mathrm{O}_{2} \mathrm{~S}$

483.1603

483.1604

13 2-\{4-[(1-Benzoylbenzimidazol-2-ylthio)methyl]-1H-

1,2,3-triazol-1-yl\}-N-( $p$-nitrophenyl)-acetamide

201-203 91

$\mathrm{C}_{26} \mathrm{H}_{23} \mathrm{~N}_{6} \mathrm{O}_{2} \mathrm{~S}$

514.1298

514.1292

503.1057

14 2-\{4-[(1-( $p$-Chlorobenzoyl)-benzimidazol-2-ylthio)

methyl]-1H-1,2,3-triazol-1-yl\}-N-phenyl-acetamide

196-198 92

$\mathrm{C}_{25} \mathrm{H}_{20} \mathrm{ClN}_{6} \mathrm{O}_{2} \mathrm{~S}$

503.1058

2-(4-\{[1-( $p$-Chlorobenzoyl)-benzimidazol-2-ylthio)

15 methyl]-1H-1,2,3-triazol-1-yl\}-N-( $p$-chlorophenyl) 206-208 acetamide

$87 \quad \mathrm{C}_{25} \mathrm{H}_{19} \mathrm{Cl}_{2} \mathrm{~N}_{6} \mathrm{O}_{2} \mathrm{~S}$

537.0667

537.0669 
2-(4-\{[1-( $p$-Chlorobenzoyl)-benzimidazol-2-ylthio]

16 methyl\}-1H-1,2,3-triazol-1-yl)- $\mathrm{N}$-( $p$-bromophenyl) 211-212 $91 \quad \mathrm{C}_{25} \mathrm{H}_{19} \mathrm{BrClN}_{6} \mathrm{O}_{2} \mathrm{~S}$

acetamide

2-(4-\{[1-( $p$-Chlorobenzoyl)-benzimidazol-2-ylthio)

17 methyl]-1H-1,2,3-triazol-1-yl\}-N-(p-nitrophenyl)

acetamide

191-193

$84 \quad \mathrm{C}_{25} \mathrm{H}_{19} \mathrm{ClN}_{7} \mathrm{O}_{4} \mathrm{~S}$

548.0908

548.0903

18 2-\{4-[(1H-Benzimidazol-2-ylthio)methyl]-1H-1,2,3-

triazol-1-yl\}-N-( $p$-methylphenyl)-acetamide

195-197 $88 \quad \mathrm{C}_{19} \mathrm{H}_{19} \mathrm{~N}_{6} \mathrm{O}_{2} \mathrm{~S}$

379.1341

379.1330

\section{Antiviral activity}

HepG2.2.15.7 cells and HepG2.2.15 clone, producing a high amount of HBV, Luc-neo\#2 and subgenomic HCV replicon cells with luciferase reporter used in testing anti-HBV and anti-HCV activity, respectively, were established and provided by Drs. Takaji Wakita and Koichi Watashi (Department of Virology II, National Institute of Infectious Diseases, Tokyo, Japan). All compounds, 4-18, were screened for their anti-HBV and anti-HCV activity using the reported method $(18,19)$. Reference inhibitors were used: lamivudine (3TC) for HBV and telaprevir (TLV) for HCV (1). Cytotoxicity was evaluated by the reduction in the number of viable cells using the tetrazolium dye method $(18,19)$. Tetracolor One ${ }^{\circledR}$ (Seikagaku Corporation, Japan) is a water-soluble tetrazolium dye that is used to determine the cell viability.

Each compound was dissolved in DMSO to produce $20 \mathrm{mmol} \mathrm{L}^{-1}$ stock solution. Each point in the dose-response curve represents the average of 3 readings.

Anti-HBV activity. - Determination of anti-HBV activity of benzimidazole derivatives was based on the inhibition of virus-induced cytopathicity. HepG2.2.15.7 cells $\left(1 \times 10^{4}\right.$ cells per well) were inoculated into a microtiter plate. After incubation for $24 \mathrm{~h}$, the cells were cultured in the presence of various concentrations of the tested compounds. After incubation for 3 and 6 days, the culture medium was replaced with fresh one containing an appropriate concentration of the compound. The cells were further incubated for 3 days. At the end of incubation, the culture supernatants were collected and examined for their HBV DNA levels by real-time PCR. The cells were examined for their viability by the tetrazolium dye method $(18,19)$.

Anti-HCV activity. - The LucNeo\#2 cells ( $5 \times 10^{3}$ cells per well) were cultured in a 96well plate in the absence of G418, an aminoglycoside commonly used to generate stable replicons for RNA viruses, such as hepatitis $C$ virus, West Nile virus, and bovine viral diarrhoea virus (BVDV), and in the presence of various concentrations of the compounds. After incubation for 3 days, the culture medium was removed, and the cells were washed once with phosphate buffer saline (PBS). Lysis buffer was added to each well, and the lysate was transferred to the corresponding well of a non-transparent 96-well plate. The luciferase activity was measured by addition of the luciferase reagent in a lucerfrease assay kit using a luminometer with automatic injectors $(18,19)$. 
Cytotoxicity assay. - LucNeo\#2 cells $\left(5 \times 10^{3}\right.$ cells per well) were cultured in a 96-well plate in the absence of G418 and in the presence of various concentrations of the compounds. After incubation for 3 days, the culture supernatants $(100 \mu \mathrm{L})$ were removed and the tetraColor ONE $(10 \mu \mathrm{L})$ was added to each well. The cells were further incubated at $37^{\circ} \mathrm{C}$ for $1 \mathrm{~h}$ the absorbance of each well was measured at $450 \mathrm{~nm}$ with a microplate reader $(18,19)$.

\section{RESULTS AND DISCUSSION}

\section{Chemistry}

In this report, hybrid molecules were prepared as illustrated in Schemes 1 and 2. Acylation of 2-(prop-2-ynyl-thio)- $1 H$-benzimidazole (1) (16) with different acyl halides in the presence of anhydrous potassium carbonate in acetone afforded 1-acyl-2-propargyl-thio derivatives $(\mathbf{2 a}-\mathbf{c})$. Structures of these compounds were established by IR, NMR and HRMS. In IR spectra, disappearance of the absorption band at $3410-3420 \mathrm{~cm}^{-1}$, along with the appearance of a strong absorption band corresponding to the carbonyl group (1680$1700 \mathrm{~cm}^{-1}$ ), confirmed $\mathrm{N}$-acylation. In ${ }^{1} \mathrm{H}$ NMR spectra, 1-acetyl-2-propyn-2-yl-thiobenzimidazole (2a) was characterized by the appearance of a singlet at $2.86 \mathrm{ppm}$, while integration
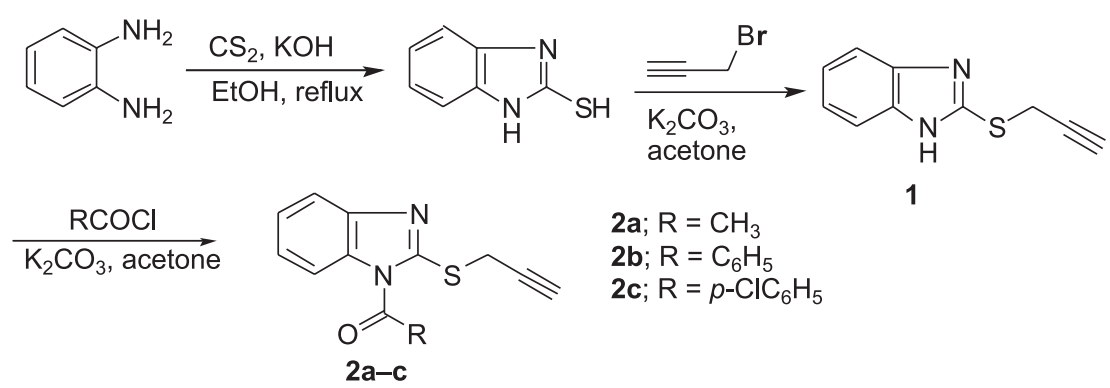

Scheme 1.<smiles>[X]c1ccc(N)cc1</smiles>

1)<smiles>O=C(Cl)CC(Cl)(Cl)Cl</smiles>

2) $\mathrm{NaN}_{3}, \mathrm{DMF}$<smiles>[R]C(=O)n1c(SCc2cn(CC(=O)Nc3ccc([X])cc3)nn2)nc2ccccc21</smiles>

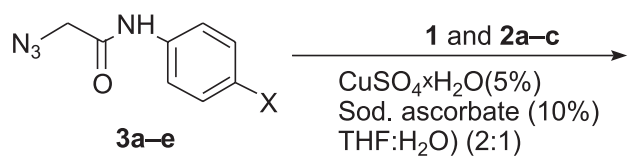

$\mathrm{X}=\mathrm{H}, \mathrm{Cl}, \mathrm{Br}, \mathrm{CH}_{3}, \mathrm{NO}_{2}$<smiles>Cc1ccc(NC(=O)Cn2cc(CSc3nc4ccccc4[nH]3)nn2)cc1</smiles>

Scheme 2. 
Table II. Spectral data of the newly synthesized compounds

\begin{tabular}{|c|c|c|c|}
\hline \multicolumn{2}{|c|}{ Compd. IR $\left(v, \mathrm{~cm}^{-1}\right)$} & $\begin{array}{l}{ }^{1} \mathrm{H} \text { NMR }\left(500 \mathrm{MHz}, \mathrm{DMSO}-d_{6}\right)(\delta, \mathrm{ppm}) \\
7.83(\mathrm{~d}, J=7.9 \mathrm{~Hz}, 1 \mathrm{H}), 7.68(\mathrm{~d}, J=7.9 \mathrm{~Hz},\end{array}$ & \multirow{2}{*}{$\begin{array}{l}{ }^{13} \mathrm{C} \text { NMR }(125 \mathrm{MHz} \\
\left.\mathrm{DMSO}-d_{6}\right)(\delta, \mathrm{ppm}) \\
170.8,154.2,144.4,133.8 \\
125.3,124.4,119.3,115.0 \\
80.9,74.7,26.9,21.0\end{array}$} \\
\hline $2 a$ & $\begin{array}{l}3307(\mathrm{spC}-\mathrm{H}), 3030 \\
1716(\mathrm{C}=\mathrm{O}), 1473,1456 \\
1371,1315(\mathrm{C}=\mathrm{C})\end{array}$ & $\begin{array}{l}7.83(\mathrm{~d}, J=7.9 \mathrm{~Hz}, 1 \mathrm{H}), 7.68(\mathrm{~d}, J=7.9 \mathrm{~Hz}, \\
1 \mathrm{H}), 7.40-7.35(\mathrm{~m}, 2 \mathrm{H}), 4.10(\mathrm{~s}, 2 \mathrm{H}), 3.22 \\
(\mathrm{~s}, 1 \mathrm{H}), 2.86(\mathrm{~s}, 3 \mathrm{H})\end{array}$ & \\
\hline $2 b$ & $\begin{array}{l}3307(\mathrm{spC}-\mathrm{H}), 3010 \\
1697(\mathrm{C}=\mathrm{O}), 1600,1473 \\
1450,1348,1313(\mathrm{C}=\mathrm{C}) \\
1193,700,648\end{array}$ & $\begin{array}{l}7.83-7.65(\mathrm{~m}, 6 \mathrm{H}), 7.31(\mathrm{~d}, J=7.2 \mathrm{~Hz}, 1 \mathrm{H}) \\
7.12(\mathrm{t}, J=7.2 \mathrm{~Hz}, 1 \mathrm{H}), 6.60(\mathrm{~d}, J=8.2 \mathrm{~Hz} \\
1 \mathrm{H}), 4.18(\mathrm{~s}, 2 \mathrm{H}), 3.7(\mathrm{~s}, 1 \mathrm{H})\end{array}$ & $\begin{array}{l}168.6,153.9,144.2,134.8 \\
134.6,133.6,130.0,129.9 \\
125.3,124.1,119.4,114.4 \\
80.6,75.0,21.5\end{array}$ \\
\hline $2 c$ & $\begin{array}{l}3307(\mathrm{spC}-\mathrm{H}), 3010 \\
1697(\mathrm{C}=\mathrm{O}), 1600,1473 \\
1450,1348,1313(\mathrm{C}=\mathrm{C}) \\
1193,700,648\end{array}$ & $\begin{array}{l}7.86(\mathrm{~d}, J=8.6 \mathrm{~Hz}, 2 \mathrm{H}), 7.75-7.69(\mathrm{~m}, \\
3 \mathrm{H}), 7.33-7.31(\mathrm{~m}, 1 \mathrm{H}), 7.18-7.16(\mathrm{~m}, 1 \mathrm{H}), \\
6.71(\mathrm{~d}, J=8.0 \mathrm{~Hz}, 1 \mathrm{H}), 4.18(\mathrm{~s}, 2 \mathrm{H}), 3.26 \\
(\mathrm{~s}, 1 \mathrm{H})\end{array}$ & $\begin{array}{l}167.6,153.8,144.2,139.7 \\
134.7,132.3,132.0,130.2 \\
125.4,124.3,119.4,114.5 \\
80.5,75.1,21.5\end{array}$ \\
\hline 4 & $\begin{array}{l}3460(\mathrm{NH}), 1706,1608 \\
(\mathrm{C}=\mathrm{O}), 1576,1544,184 \\
1475(\mathrm{C}=\mathrm{C}), 715,742\end{array}$ & $\begin{array}{l}10.49(\mathrm{~s}, 1 \mathrm{H}), 8.18(\mathrm{~s}, 1 \mathrm{H}), 7.85(\mathrm{~d}, J=8.2 \\
\mathrm{Hz}, 1 \mathrm{H}), 7.72(\mathrm{~d}, J=8.0 \mathrm{~Hz}, 1 \mathrm{H}), 7.60(\mathrm{~d}, \\
J=8.2 \mathrm{~Hz}, 2 \mathrm{H}), 7.39-7.35(\mathrm{~m}, 4 \mathrm{H}), 7.12 \\
(\mathrm{t}, J=8.2 \mathrm{~Hz}, 1 \mathrm{H}), 5.34(\mathrm{~s}, 2 \mathrm{H}), 4.62(\mathrm{~s}, 2 \mathrm{H}), \\
2.86(\mathrm{~s}, 3 \mathrm{H})\end{array}$ & $\begin{array}{l}170.7,165.2,155.1,144.5, \\
143.4,139.4,133.9,129.9, \\
126.5,125.2,124.7,124.3, \\
120.2,119.4,115.1,53.2,27.5 \\
27.0\end{array}$ \\
\hline 5 & $\begin{array}{l}\text { IR3430 (NH), 1710, } 1690 \\
(\mathrm{C}=\mathrm{O}), 1607,1581,1548, \\
1456(\mathrm{C}=\mathrm{C}), 840\end{array}$ & $\begin{array}{l}10.63(\mathrm{~s}, 1 \mathrm{H}), 8.18(\mathrm{~s}, 1 \mathrm{H}), 7.85(\mathrm{~d}, J=8.0 \\
\mathrm{Hz}, 1 \mathrm{H}), 7.70(\mathrm{~d}, J=6.9 \mathrm{~Hz}, 1 \mathrm{H}), 7.62(\mathrm{~d}, J \\
=6.9 \mathrm{~Hz}, 2 \mathrm{H}), 7.43-7.35(4 \mathrm{H}, \mathrm{m}), 5.35(\mathrm{~s}, \\
2 \mathrm{H}), 4.69(\mathrm{~s}, 2 \mathrm{H}), 2.86(\mathrm{~s}, 3 \mathrm{H})\end{array}$ & $\begin{array}{l}170.8,165.4,155.1,144.6, \\
143.5,133.9,130.2,129.9, \\
128.4,126.5,125.3,124.4, \\
121.8,119.5,115.2,53.2,27.6, \\
27.1\end{array}$ \\
\hline 6 & $\begin{array}{l}3445(\mathrm{NH}), 1704,1686 \\
(\mathrm{C}=\mathrm{O}), 1604,1582,1554, \\
1485(\mathrm{C}=\mathrm{C}), 712,743\end{array}$ & $\begin{array}{l}10.63(\mathrm{~s}, 1 \mathrm{H}), 8.18(\mathrm{~s}, 1 \mathrm{H}), 7.85-7.84(\mathrm{~m}, \\
3 \mathrm{H}), 7.71(\mathrm{~s}, 1 \mathrm{H}), 7.58-7.54(\mathrm{~m}, 2 \mathrm{H}) \\
7.40-7.34(\mathrm{~m}, 2 \mathrm{H}), 5.35(\mathrm{~s}, 2 \mathrm{H}), 4.62(\mathrm{~s} \\
2 \mathrm{H}), 2.86(\mathrm{~s}, 3 \mathrm{H})\end{array}$ & $\begin{array}{l}\text { 170.5, 165.3, 154.9, 144.5, } \\
143.3,138.6,133.8,132.6, \\
126.3,125.2,124.2,122.1, \\
119.3,116.3,115.0,53.1,27.4 \\
26.9\end{array}$ \\
\hline 7 & $\begin{array}{l}3435(\mathrm{NH}), 1696,1687 \\
(\mathrm{C}=\mathrm{O}), 1608,1554,1492, \\
1455(\mathrm{C}=\mathrm{C}), 830\end{array}$ & $\begin{array}{l}10.4(\mathrm{~s}, 1 \mathrm{H}), 8.17(\mathrm{~s}, 1 \mathrm{H}), 7.85(\mathrm{~d}, J=7.6 \\
\mathrm{Hz}, 1 \mathrm{H}), 7.72(\mathrm{~d}, J=6.9 \mathrm{~Hz}, 2 \mathrm{H}), 7.47(\mathrm{~d} \\
J=8.2 \mathrm{~Hz}, 1 \mathrm{H}), 7.39-7.35(\mathrm{~m}, 2 \mathrm{H}), 7.16 \\
(\mathrm{~d}, J=8.2 \mathrm{~Hz}, 2 \mathrm{H}), 5.32(\mathrm{~s}, 2 \mathrm{H}), 4.62(\mathrm{~s} \\
2 \mathrm{H}), 2.86(\mathrm{~s}, 3 \mathrm{H}), 2.92(\mathrm{~s}, 3 \mathrm{H})\end{array}$ & $\begin{array}{l}\text { 170.7, 164.9, 155.1, 144.5, } \\
\text { 143.3, 136.8, 133.9, } 133.7 . \\
\text { 130.2, 126.4, 125.2, 124.3, } \\
\text { 120.2, 119.4, 115.1, 53.3, 27.5, } \\
\text { 27.0, } 21.4\end{array}$ \\
\hline 8 & $\begin{array}{l}3435(\mathrm{NH}), 1710,1687 \\
(\mathrm{C}=\mathrm{O}), 1607,1556,1518 \\
1456(\mathrm{C}=\mathrm{C}), 837\end{array}$ & $\begin{array}{l}11.10(\mathrm{~s}, 1 \mathrm{H}), 8.27(\mathrm{~d}, J=9.2 \mathrm{~Hz}, 2 \mathrm{H}), 8.20 \\
(\mathrm{~s}, 1 \mathrm{H}), 7.86-7.84(\mathrm{~m}, 3 \mathrm{H}), 7.71(\mathrm{~d}, J=7.6 \\
\mathrm{Hz}, 1 \mathrm{H}), 7.40-7.35(\mathrm{~m}, 2 \mathrm{H}), 5.43(\mathrm{~s}, 2 \mathrm{H}), \\
4.62(\mathrm{~s}, 2 \mathrm{H}), 2.77(\mathrm{~s}, 3 \mathrm{H})\end{array}$ & $\begin{array}{l}170.7,166.3,163.3,155.0 \\
145.4,144.5,143.5,133.9, \\
126.5,126.1,125.2,124.3 \\
120.0,119.4,115.1,53.3,27.5 \\
27.0\end{array}$ \\
\hline 9 & $\begin{array}{l}3405(\mathrm{NH}), 1702,1671 \\
(\mathrm{C}=\mathrm{O}), 1615,1589,1472 \\
1453(\mathrm{C}=\mathrm{C}), 712,743\end{array}$ & $\begin{array}{l}10.49(\mathrm{~s}, 1 \mathrm{H}), 8.20(\mathrm{~s}, 1 \mathrm{H}), 7.82-7.80(\mathrm{~m}, \\
1 \mathrm{H}), 7.73-7.72(\mathrm{~m}, 2 \mathrm{H}), 7.65(\mathrm{t}, J=7.9 \mathrm{~Hz}, \\
1 \mathrm{H}), 7.60-7.59(\mathrm{~m}, 3 \mathrm{H}), 7.37-7.32(\mathrm{~m}, 3 \mathrm{H}), \\
7.42(\mathrm{~d}, J=8.0 \mathrm{~Hz}, 1 \mathrm{H}), 7.32(\mathrm{t}, J=8.0 \mathrm{~Hz}, \\
1 \mathrm{H}), 7.14-7.10(\mathrm{~m}, 2 \mathrm{H}), 5.34(\mathrm{~s}, 2 \mathrm{H}), 4.69 \\
(\mathrm{~s}, 2 \mathrm{H})\end{array}$ & $\begin{array}{l}168.7,165.1,154.7,144.3 \\
143.2,139.3,134.9,134.6 \\
133.7,130.1,130.0,128.4 \\
126.5,125.2,124.7,124.1 \\
120.2,119.4,114.4,53.2,27.9\end{array}$ \\
\hline
\end{tabular}


10

$3425(\mathrm{NH}), 1710,1668$ $(\mathrm{C}=\mathrm{O}), 1626,1593,1529$, 1474 (C=C), 837

3435 (NH), 1710, 1669

11 (C=O), 1591, 1518, 1495, 1466 (C=C), 842

3415 (NH), 1704, 1670

12

13

14

15

$3420(\mathrm{NH}), 1710,1682$ $(\mathrm{C}=\mathrm{O}), 1622,1592,1552$, $1452(\mathrm{C}=\mathrm{C}), 842$

3425 (NH), 1704, 1680 (C=O), 1626, 1593, 1558, 1475 ( $\mathrm{C}=\mathrm{C}), 830$

3420 (NH), 1714, 1670 $(\mathrm{C}=\mathrm{O}), 1616,1593,1561$, $1451(\mathrm{C}=\mathrm{C}), 834$

16

3430 (NH), 1704, 1674 $(\mathrm{C}=\mathrm{O}), 1613,1593,1559$, $1486(\mathrm{C}=\mathrm{C}), 837$

3445 (NH), 1712, 1675

17 $1453(\mathrm{C}=\mathrm{C}), 837$

$10.64(\mathrm{~s}, 1 \mathrm{H}), 8.19(\mathrm{~s}, 1 \mathrm{H}), 7.82-7.80(\mathrm{~m}, \quad 168.8,165.4,154.8,144.4$, $4 \mathrm{H}), 7.72(\mathrm{~d}, J=8.0 \mathrm{~Hz}, 1 \mathrm{H}), 7.66-7.62(\mathrm{~m}, 143.3,138.4,135.0,134.7$, $4 \mathrm{H}), 7.42(\mathrm{~d}, J=7.9 \mathrm{~Hz}, 1 \mathrm{H}), 7.42(\mathrm{~d}, J=8.0133 .8,130.2,130.1,129.9$, $\mathrm{Hz}, 1 \mathrm{H}), 7.32(\mathrm{t}, J=8.0 \mathrm{~Hz}, 1 \mathrm{H}), 7.13(\mathrm{t}, J=128.4,126.6,125.3,124.2$, $8.0 \mathrm{~Hz}, 1 \mathrm{H}), 5.35(\mathrm{~s}, 2 \mathrm{H}), 4.69(\mathrm{~s}, 2 \mathrm{H}) \quad 121.8,119.5,114.4,53.3,28.0$

$10.63(\mathrm{~s}, 1 \mathrm{H}), 8.19(\mathrm{~s}, 1 \mathrm{H}), 7.82-7.80(\mathrm{~m}, \quad 168.7,165.4,154.7,144.3$, $3 \mathrm{H}), 7.72(\mathrm{~d}, J=8.2 \mathrm{~Hz}, 1 \mathrm{H}), 7.66-7.64 \quad 143.2,138.7,134.9,134.6$, (m, 2H), 7.58-7.54 (m, 4H), 7.33-7.11 (m, 133.7, 132.7, 130.1, 130.0, $2 \mathrm{H}), 6.62(\mathrm{~d}, J=8.2 \mathrm{~Hz}, 1 \mathrm{H}), 5.35(\mathrm{~s}, 2 \mathrm{H}), 126.5,125.2,124.1,122.1$, $4.69(\mathrm{~s}, 2 \mathrm{H})$ $119.4,116.4,114.4,53.2,27.9$ $10.41(\mathrm{~s}, 1 \mathrm{H}), 8.18(\mathrm{~s}, 1 \mathrm{H}), 7.81-7.80(\mathrm{~m}, \quad 168.7,164.9,154.7,144.3$, $2 \mathrm{H}), 7.72(\mathrm{~d}, J=7.9 \mathrm{~Hz}, 1 \mathrm{H}), 7.65(\mathrm{t}, J=7.9143 .1,136.8,134.9,134.6$, $\mathrm{Hz}, 3 \mathrm{H}), 7.48(\mathrm{~d}, J=7.9 \mathrm{~Hz}, 1 \mathrm{H}), 7.32(\mathrm{t}, J$ 133.7, 133.6, 130.2, 130.1, $=7.6 \mathrm{~Hz}, 1 \mathrm{H}), 7.17-7.12(\mathrm{~m}, 3 \mathrm{H}), 6.62(\mathrm{~d}, J$ 130.0, 126.5, 125.2, 124.1, $=8.0 \mathrm{~Hz}, 1 \mathrm{H}), 5.32(\mathrm{~s}, 2 \mathrm{H}), 4.69(\mathrm{~s}, 2 \mathrm{H}), 120.2,119.4,114.4,53.2,27.9$, $2.29(\mathrm{~s}, 3 \mathrm{H})$ 21.4

$11.10(\mathrm{~s}, 1 \mathrm{H}), 8.27(\mathrm{~d}, J=9.0 \mathrm{~Hz}, 2 \mathrm{H}), 8.21 \quad 168.7,166.3,154.7,145.4$, (s, 1H), 7.86-7.70 (m, 4H), $7.72(\mathrm{~d}, J=7.6 \quad 144.3,143.5,143.3,134.9$, $\mathrm{Hz}, 1 \mathrm{H}), 7.65(\mathrm{t}, J=8.0 \mathrm{~Hz}, 2 \mathrm{H}), 7.32(\mathrm{t}, J=134.7,133.7,130.1,130.0$, $7.6 \mathrm{~Hz}, 1 \mathrm{H}), 7.12(\mathrm{t}, J=7.6 \mathrm{~Hz}, 2 \mathrm{H}), 6.62(\mathrm{~d}, 126.5,126.0,125.2,124.1$, $J=8.0 \mathrm{~Hz}, 1 \mathrm{H}), 5.44(\mathrm{~s}, 2 \mathrm{H}), 4.70(\mathrm{~s}, 2 \mathrm{H}) \quad 120.0,119.4,114.4,53.3,27.9$

10.49 (s, 1H), 8.19 (s, 1H), $7.85(\mathrm{~d}, J=8.2 \quad 167.7,165.1,154.6,144.3$, $\mathrm{Hz}, 2 \mathrm{H}), 7.73(\mathrm{~d}, J=8.2 \mathrm{~Hz}, 3 \mathrm{H}), 7.60(\mathrm{~d}, 143.1,139.5,139.3,134.8$, $J=8.0 \mathrm{~Hz}, 2 \mathrm{H}), 7.38-7.32(\mathrm{~m}, 3 \mathrm{H}), 7.12(\mathrm{t}, 132.4,132.2,130.2,129.9$, $J=8.2 \mathrm{~Hz}, 1 \mathrm{H}), 6.73(\mathrm{~d}, J=8.2 \mathrm{~Hz}, 1 \mathrm{H}), \quad 126.5,125.3,124.7,124.2$, $5.34(\mathrm{~s}, 2 \mathrm{H}), 4.69(\mathrm{~s}, 2 \mathrm{H})$ $120.1,119.4,114.5,53.2,27.9$

10.59 (s, 1H), $8.14(\mathrm{~s}, 1 \mathrm{H}), 7.80(\mathrm{~d}, J=8.6$ $\mathrm{Hz}, 2 \mathrm{H}), 7.67(\mathrm{~d}, J=8.6 \mathrm{~Hz}, 2 \mathrm{H}), 7.57(\mathrm{~d}$, $J=8.5 \mathrm{~Hz}, 3 \mathrm{H}), 7.37(\mathrm{t}, J=8.5 \mathrm{~Hz}, 2 \mathrm{H})$, $7.28(\mathrm{t}, J=7.5 \mathrm{~Hz}, 1 \mathrm{H}), 7.12(\mathrm{t}, J=7.5 \mathrm{~Hz}$, $1 \mathrm{H}), 6.67(\mathrm{t}, J=8.2 \mathrm{~Hz}, 1 \mathrm{H}), 5.30(\mathrm{~s}, 2 \mathrm{H})$ $4.64(\mathrm{~s}, 2 \mathrm{H})$

$167.8,165.3,154.6,144.3$, $143.2,139.5,138.3,134.8$ $132.4,132.2,130.2,129.8$ $129.3,126.5,125.3,124.2$ $121.7,119.4,114.5,53.2,27.9$

$10.59(\mathrm{~s}, 1 \mathrm{H}), 8.16(\mathrm{~s}, 1 \mathrm{H}), 7.82(\mathrm{~d}, J=8.2 \quad 167.2,164.9,154.1,143.8$, $\mathrm{Hz}, 2 \mathrm{H}), 7.70(\mathrm{~d}, \mathrm{~J}=8.2 \mathrm{~Hz}, 2 \mathrm{H}), 7.56-7.50142 .7,139.0,138.2,134.3$, $(\mathrm{m}, 2 \mathrm{H}), 7.58-7.54(\mathrm{~m}, 3 \mathrm{H}), 7.30(\mathrm{t}, J=8.2 \quad 132.2,131.9,131.7,129.7$, $\mathrm{Hz}, 1 \mathrm{H}), 7.14(\mathrm{t}, J=8.2 \mathrm{~Hz}, 1 \mathrm{H}), 6.70(\mathrm{~d}, J$ 126.0, 124.8, 123.7, 121.6, $=8.2 \mathrm{~Hz}, 1 \mathrm{H}), 5.32(\mathrm{~s}, 2 \mathrm{H}), 4.66(\mathrm{~s}, 2 \mathrm{H}) \quad 118.9,115.9,114.0,52.7,27.5$

$11.10(\mathrm{~s}, 1 \mathrm{H}), 8.27(\mathrm{~d}, J=9.3 \mathrm{~Hz}, 2 \mathrm{H}), 8.21167 .7,166.3,154.6,145.4$, (s, 1H), 7.86-7.84 (m, 4H), 7.74-7.72 (m, 144.3, 143.5, 143.2, 139.5, $3 \mathrm{H}), 7.33(\mathrm{t}, J=7.2 \mathrm{~Hz}, 1 \mathrm{H}), 7.17(\mathrm{t}, J=7.2134 .8,132.4,132.1,130.2$, $\mathrm{Hz}, 1 \mathrm{H}), 6.72(\mathrm{~d}, J=8.2 \mathrm{~Hz}, 1 \mathrm{H}), 5.44(\mathrm{~s}, 126.5,126.1,125.3,124.2$, $2 \mathrm{H}), 4.70(\mathrm{~s}, 2 \mathrm{H})$ $120.0,119.4,114.5,53.3,27.9$

$163.8,149.7,143.6,143.2$, $3415(\mathrm{NH}), 1673(\mathrm{C}=\mathrm{O}), 10.36(\mathrm{~s}, 1 \mathrm{H}), 8.10(\mathrm{~s}, 1 \mathrm{H}), 7.52-7.43(\mathrm{~m}$, 
of signals at $\delta$ 8-7 ppm confirmed the structures of 1-benzoyl-2-propyn-2-yl-thiobenzimidazole (2b) and 1-p-chlorobenzoyl-2-propyn-2-yl-thiobenzimidazole (2c). In ${ }^{13} \mathrm{C} N M R$, the presence of a signal in the region $\delta 170-168 \mathrm{ppm}$ confirmed acylation. Further, reacting compounds $\mathbf{2 a - c}$ with azido compounds $3 \mathbf{a}-\mathbf{e}$ (17) using the $\mathrm{Cu}$ alkyne-azide cycloaddition (CuAAC) (20-23) provided the target compounds $\mathbf{4} \mathbf{- 1 8}$ in excellent yields (85-90 \%). In particular, in each cycloadduct, the proton associated with the 1,2,3-triazole moiety was identified as a singlet with $\delta$ between 8.18 and $8.20 \mathrm{ppm}$. The structures of all the new compounds were confirmed also by mass spectral data (ESI-HRMS) where the found and calculated mass units are identical up to 4 decimal digits. All physical and spectral data of the new compounds are given in Tables I and II.

\section{Antiviral activity}

Target compounds were designed based on the available data (Fig. 1) (8-10). Benzimidazoles substituted at position-2 with amide, urea and/or heterocycle showed good anti-HCV and/or anti-HBV activity. Accordingly, and in search of a potent antiviral agent, 2-mercaptobenzimidazoles were linked to anilides with a triazole ring. It was proposed that the triazole ring along with sulphur atom would improve the activity of lead molecules. All synthesized compounds considered in this work were evaluated for antiviral activity against $\mathrm{HBV}$ and $\mathrm{HCV}$.

Cytotoxicity was evaluated in parallel with the antiviral activity. The strategy followed in the antiviral screening study is based on using two concentrations ( 1 and $10 \mu \mathrm{mol} \mathrm{L}^{-1}$ ) of each of the test compounds. Test compounds showed no significant activity against HBV and weak activity toward HCV (Figs. 2a and b). Among the tested compounds, 2-\{4-[(1-benzoylbenzimidazol-2-ylthio)methyl]-1H-1,2,3-triazol-1-yl\}-N-( -nitrophenyl)-acetamide (13) and 2-(4-\{[1-( $p$-chlorobenzoyl)benzimidazol-2-ylthio)methyl]-1H-1,2,3-triazol-1-yl\}-N-( $p$ nitrophenyl)acetamide (17) showed significant activity and were subjected to further investigation at various concentrations in comparison with the reference drug telaprevir (TLV). Judging by the results, fifty percent effective concentrations $\left(E C_{50}\right)$ of $\mathrm{HCV}$ inhibition for compounds 13 and 17 were 7.8 and $7.6 \mu \mathrm{mol} \mathrm{L}^{-1}$, respectively, and the $50 \%$ cytotoxic concentrations $\left(\mathrm{CC}_{50}\right)$ were 16.9 and $21.1 \mu \mathrm{mol} \mathrm{L}^{-1}$, thus resulting in selectivity indices of 2-3 (Fig. 3). For telaprevir see also ref. 24. Consulting the inhibitory values for the compounds as anti-HCV agents, we found that the anilide part having an electron withdrawing group, notably nitro group, showed comparatively better activity than other substituents. The results could be explained according to the Topliss scheme $(25,26)$, which starts with the unsubstituted phenyl. In this work, 2-\{4-[(1-acetylbenzimidazol-2-yl-thio)methyl]1H-1,2,3-triazol-1-yl\}- $N$-( $p$-chlorophenyl)-acetamide (5) with $p$-chlorophenyl showed higher affinity than 2-\{4-[(1-acetylbenzimidazol-2-yl-thio)methyl]-1H-1,2,3-triazol-1-yl\}- $N$-phenylacetamide (4) with unsubstituted phenyl. For the same reason, 2-\{4-[(1-benzoylbenzimid azol-2-yl-thio)methyl]-1 H-1,2,3-triazol-1-yl\}- $N$-( $p$-chlorophenyl)acetamide $\quad(10)$ and 2-(4-\{[1-( $p$-chlorobenzoyl)benzimidazol-2-yl-thio)methyl]-1H-1,2,3-triazol-1-yl\}- $N$-( $p$-chlorophenyl)acetamide (15) showed higher activity than 2-\{4-[(1-benzoylbenzimidazol-2-ylthio)methyl]-1 $H-1,2,3$-triazol-1-yl\}-N-phenylacetamide (9) and 2-\{4-[(1-( $p$-chlorobenzoyl) benzimidazol-2-yl-thio)methyl]-1H-1,2,3-triazol-1-yl\}- $N$-phenyl-acetamide (14), which most probably can be attributed to a positive $\pi$-effect, a positive $\alpha$-effect or to a combination of both. Since the potency of 2-\{4-[(1-benzoylbenzimidazol-2-yl-thio)methyl]-1H-1,2,3-triazol1 -yl\}-N-( $p$-methylphenyl)-acetamide (12) with $p$-methylphenyl was lower than that of 

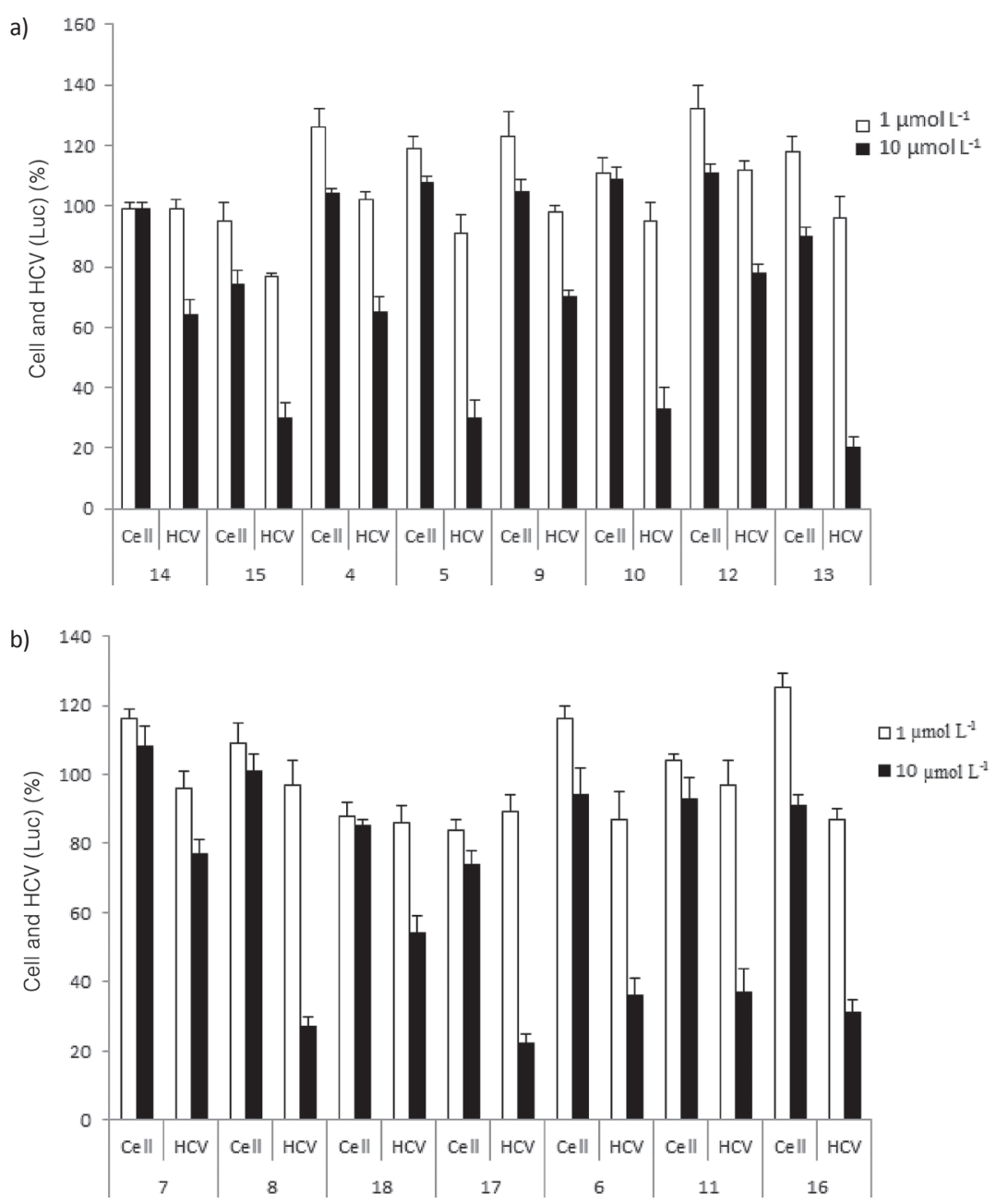

Fig. 2. Anti-HCV and cytotoxicity of compounds: a) 4, 5, 9, 10, 12-15, and b) compounds $6-8, \mathbf{1 1}, \mathbf{1 6}-\mathbf{1 8}$ $(\mathrm{SD}, n=3)$.

2-\{4-[(1-(p-chlorobenzoyl)benzimidazol-2-yl-thio)methyl]-1H-1,2,3-triazol-1-yl\}-N-phenylacetamide (14), it may be concluded that the positive $\pi$-effect is dominant. Accordingly, $p$-nitrophenyl derivatives were synthesized [2-\{4-[(1-acetylbenzimidazol-2-yl-thio)methyl]1H-1,2,3-triazol-1-yl\}-N-( $p$-nitrophenyl)-acetamide (8), 2-\{4-[(1-benzoylbenzimidazol-2-ylthio)methyl]-1H-1,2,3-triazol-1-yl\}- $N$-( $p$-nitrophenyl)-acetamide (13) and 2-(4-\{[1-( $p$-chlorobenzoyl)benzimidazol-2-yl-thio)methyl]-1 $H$-1,2,3-triazol-1-yl\}- $N$-( $p$-nitrophenyl)acetamide (17). As expected, the latter compounds (13 and 17) showed the highest inhibitory activity among all the synthesized compounds (Fig. 3). 


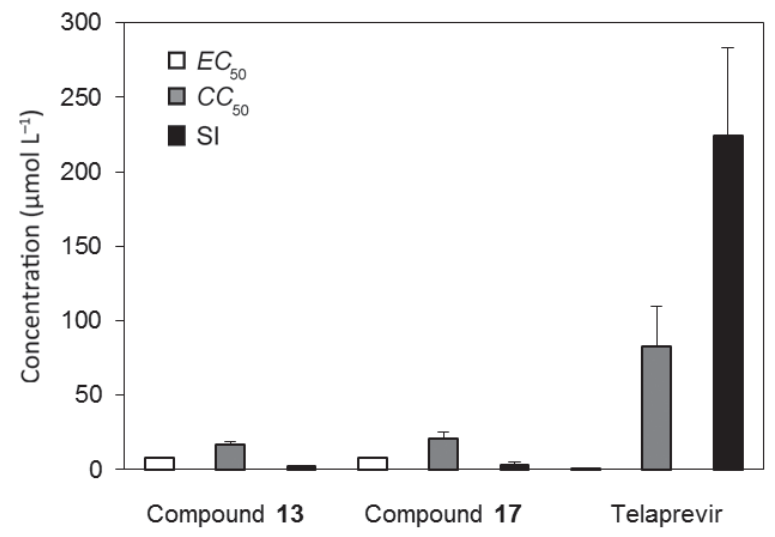

Fig. 3. Fifty percent effective concentration of HCV inhibition (EC50), $50 \%$ cytotoxic concentration (CC50) and CC50 to EC50 ratio (selectivity index, SI) for compounds $\mathbf{1 3}$ and $\mathbf{1 7}$ and reference drug telaprevir (SD, $n=3)$.

\section{CONCLUSIONS}

In summary, we have described the synthesis of a series of hybrid molecules, which combined two heterocycles (benzimidazole and 1,2,3-triazole) in excellent yields using simple, efficient, and fast routes by taking advantage of the click chemistry strategy. These benzimidazole/triazoles were tested against HBV and HCV. Among compounds reported in this study, compounds 2-\{4-[(1-benzoylbenzimidazol-2-yl-thio)methyl]-1H-1,2,3-triazol-1-yl\}-N-( $p$-nitrophenyl)acetamide (13) and 2-(4-\{[1-( $p$-chlorobenzoyl)benzimidazol-2-ylthio)methyl]-1H-1,2,3-triazol-1-yl\}- $N$-( $p$-nitrophenyl)acetamide (17) showed significant activity against $\mathrm{HCV}$.

Acknowledgements. - This study was supported by the Faculty of Pharmacy, Assiut University, and the Faculty of Pharmacy, Al-Azhar University at Assuit. We are also grateful to Dr. Masaaki Toyama, Division of Antiviral Chemotherapy, Center for Chronic Viral Diseases, Kagoshima University, Japan, for helping is with testing the antiviral activity.

\section{REFERENCES}

1. J. S. Au and P. J. Pockros, Novel therapeutic approaches for hepatitis C, Clin. Pharmacol. Ther. 95 (2014) 78-88; DOI: 10.1038/clpt.2013.206.

2. M. P. Manns, J. G. McHutchison, S. C. Gordon, V. K. Rustgi, M. Shiffman, R. Reindollar, Z. D. Goodman, K. Koury, M. Ling and J. K. Albrecht, Peginterferon alfa-2b plus ribavirin compared with interferon alfa-2b plus ribavirin for initial treatment of chronic hepatitis $\mathrm{C}$ : a randomised trial, Lancet 358 (2001) 958-965.

3. M. W. Fried, M. L. Shiffman, K. R. Reddy, C. Smith, G. Marinos, F. L. Gonçales Jr., D. Häussinger, M. Diago, G. Carosi, D. Dhumeaux, A. Craxi, A. Lin, J. Hoffman and J. Yu, Peginterferon alfa-2a plus ribavirin for chronic hepatitis C virus infection, N. Engl. J. Med. 347 (2002) 975-982; DOI: 10.1056/NEJMoa020047. 
4. Y. Bansal and O. Silakari, The therapeutic journey of benzimidazoles: A review, Bioorg. Med. Chem. 20 (2012) 6208-6236; DOI: 10.1016/j.bmc.2012.09.013.

5. V. Boido, G. Paglietti, M. Tonelli and G. Vitale, Non-nucleoside Benzimidazoles as Antiviral Drugs Against HCV and RSV Infections, in RNA-Viruses. Enzymatic and Receptoral Inhibitors (Ed. A. Carta), Research Signpost, Kerala 2009, pp. 41-93.

6. B. A. Katz, J. Clark, J. Finer-Moore, T. Jenkins, C. Johnson, M. Ross, C. Luong, W. Moore and R. Stroud, Design of potent selective zinc-mediated serine protease inhibitors, Nature 391 (1998) 608-612; DOI: 10.1038/35422.

7. D. Sperandio, A. R. Gangloff, J. Litvak, R. Goldsmith, J. M. Hataye, V. R. Wang, E. J. Shelton, K. Elrod, J. W. Janc, J. M. Clark, K. Rice, S. Weinheimer, K. Yeung, N. A. Meanwell, D. Hernandez, A. J. Staab, B. L. Venables and J. R. Spencer, Highly potent non-peptidic inhibitors of the HCV NS3/NS4A serine protease, Bioorg. Med. Chem. Lett. 12 (2002) 3129-3133; DOI: 10.1016/S0960-894X(02)00680-7.

8. H. I. El Diwani, H. T. Abdel-Mohsen, I. Salama, F. A.-F. Ragab, M. M. Ramla, S. A. Galal, M. M. Abdalla, A. Abdel-Wahab and M. A. El Demellawy, Synthesis, molecular modeling, and biological evaluation of novel benzimidazole derivatives as inhibitors of hepatitis C virus RNA replication, Chem. Pharm. Bull. 62 (2014) 856-866; DOI: 10.1248/cpb.c13-01009.

9. L. Garuti, M. Roberti and G. Gentilomi, Synthesis and antiviral assays of some 2-substituted benzimidazole-N-carbamates, Farmaco 55 (2000) 35-39; DOI: 10.1016/S0014-827X(99)00117-2.

10. S. Budow, M. Kozlowska, A. Gorska, Z. Kazimierczuk, H. Eickmeier, P. La Colla, G. Gosselin and F. Seela, Substituted benzimidazole: antivral activity and synthesis of nucleosides, ARKIVOC iii (2009) 225-250; DOI: 10.3998/ark.5550190.0010.319.

11. M. F. Tonelli, F. Novelli, B. Tasso, I. Vazzana, A. Sparatore, V. Boido, F. Sparatore, P. La Colla, G. Sanna, G. Giliberti, B. Busonera, P. Farci, C. Ibba and R. Loddo, Antiviral activity of benzimidazole derivatives. III. Novel anti-CVB-5, anti-RSV and anti-Sb-1 agents, Bioorg. Med. Chem. 22 (2014) 4893-4909; DOI: 10.1016/j.bmc.2014.06.043.

12. Y. Saito, V. Escuret, D. Durantel, F. Zoulim, R. F. Schinazic and L. A. Agrofoglio, Synthesis of 1,2,3-triazolo-carbanucleoside analogues of ribavirin targeting an HCV in replicon, Bioorg. Med. Chem. 11 (2003) 3633-3639; DOI: 10.1016/S0968-0896(03)00349-3.

13. F. D. Da Silva, M. C. B. V. de Souza, I. I. P. Frugulhetti, H. C. Castro, S. L. D Souza, T. M. L. de Souza, D. Q. Rodrigues, A. M. T. Souza, P. A. Abreu, F. Passamani, C. R. Rodrigues and V. F. Ferreira, Synthesis, HIV-RT inhibitory activity and SAR of 1-benzyl-1H-1,2,3-triazole derivatives of carbohydrates, Eur. J. Med. Chem. 44 (2009) 373-383; DOI: 10.1016/j.ejmech.2008.02.047.

14. D. K. Mohapatra, P. K. Maity, M. Shabab and M. I. Khan, Click chemistry based rapid one-pot synthesis and evaluation for protease inhibition of new tetracyclic triazole fused benzodiazepine derivatives, Bioorg. Med. Chem. 19 (2009) 5241-5245; DOI: 10.1016/j.bmcl.2009.06.107.

15. T. O. Olomola, R. Klein, K. A. Lobb, Y. Sayed and P. T. Kaye, Synthesis and evaluation of coumarin derivatives as potential dual-action HIV-1 protease and reverse transcriptase inhibitors, Bioorg. Med. Chem. 21 (2013) 1964-1971; DOI: 10.1016/j.bmc.2013.01.025.

16. M. M. Heravi, A. Keivanloo, M. Rahimizadeh, M. Bakavoli and M. Ghassemzadeh, Pd-Cu catalyzed heterocyclization during Sonogashira coupling: synthesis of 3-benzylthiazolo[3,2-a]benzimidazole, Tetrahedron Lett. 45 (2004) 5747-5749; DOI: 10.1016/j.tetlet.2004.05.094.

17. K.-C. Tiew, D. Dou, T. Teramoto, H. Lai, K. R. Alliston, G. H. Lushington, R. Padmanabhan and W. C. Groutas, Inhibition of Dengue virus and West Nile virus proteases by click chemistry-derived benz[d] isothiazol-3(2H)-one derivatives, Bioorg. Med. Chem. 20 (2012) 1213-1221; DOI: 10.1016/j.bmc.2011.12.047.

18. C. Baba, K. Yanagida, T. Kanzaki and M. Baba, Colorimetric lactate dehydrogenase (LDH) assay for evaluation of antiviral activity against bovine viral diarrhoea virus (BVDV) in vitro, Antivir. Chem. Chemother. 16 (2005) 33-39; DOI: 10.1016/j.biomaterials.2014.01.054. 
19. K. Sako, H. Aoyama, S. Sato, Y. Hashimoto and M. Baba, $\gamma$-carboline derivatives with anti-bovine viral diarrhea virus (BVDV) activity, Bioorg. Med. Chem. 16 (2008) 3780-3790; DOI: 10.1016/j.bmc.2008.01.052.

20. H. C. Kolb, M. G. Finn and K. B. Sharpless, Click chemistry: Diverse chemical function from a few good reactions, Angew. Chem. Int. Ed. 40 (2001) 2004-2021; DOI: 10.1002/1521-3773(20010601)40: $11<2004::$ AID-ANIE2004>3.0.CO;2-5.

21. J. E. Moses and A. D. Moorhouse, The growing applications of click chemistry, Chem. Soc. Rev. 36 (2007) 1249-1262; DOI: 10.1039/b613014n.

22. S. G. Agalave, S. R. Maujan and V. S. Pore, Click chemistry: 1,2,3-Triazoles as pharmacophores, Chem. Asian J. 6 (2011) 2696-2718; DOI: 10.1002/asia.201100432.

23. J.-F. Lutz and Z. Zarafshani, Efficient construction of therapeutics, bioconjugates, biomaterials and bioactive surfaces using azide-alkyne "click" chemistry, Adv. Drug Deliv. Rev. 60 (2008) 958-970; DOI: 10.1016/j.addr.2008.02.004.

24. K. Lin, R. B. Perni, A. D. Kwong and c. Lin, VX-950, a novel hepatitis C virus (HCV) NS3-4A protease inhibitor, exhibits potent antiviral activities in HCV replicon cells, Antimicrob. Agents. Cli. 50 (2006) 1813-1822; DOI: 10.1128/AAC.50.5.1813-1822.2006.

25. J. G. Topliss, Utilization of operational schemes for analog synthesis in drug design, J. Med. Chem. 15 (1972) 1006-1011; DOI: 10.1021/jm00280a002.

26. J. G. Topliss, A manual method for applying the Hansch approach to drug design, J. Med. Chem. 20 (1977) 463-469; DOI: 10.1021/jm00214a001. 